\title{
Perfil epidemiológico das diferentes fraturas de fêmur de pacientes internados em um hospital do norte de Minas Gerais
}

\author{
Epidemiological Profile of different femur fractures of patients admitted to a hospital in the \\ north of Minas Gerais
}
Perfil epidemiológico de diferentes fracturas de fémur de pacientes ingresados en un hospital del norte de Minas Gerais

Rafael Oliveira Rocha ${ }^{1 *}$, Luís Roberto Félix Alkmim¹, Alexander Rocha Siqueira ${ }^{2}$, Mariza Dias Xavier $^{2}$, Samuel de Paiva Oliveira², Claudiana Donato Bauman².

\section{RESUMO}

Objetivo: Analisar o perfil epidemiológico, sociodemográfico e clínico das fraturas de quadril e femoral de pacientes internados em um Hospital do Norte de Minas Gerais. Métodos: Trata-se de um estudo epidemiológico, com delineamento documental, retrospectivo, descritivo e analítico conduzido com 144 pacientes que deram entrada no hospital com fratura de Fêmur e Quadril entre o período de janeiro de 2018 a janeiro de 2020. Para obtenção dos dados, foi utilizado um formulário sociodemográfico (idade e sexo) e prontuário para a evolução (retrospectiva). Os dados foram analisados através da estatística descritiva utilizando-se o programa IBM SPSS Statistics. O projeto foi aprovado pelo Comitê de Ética em Pesquisa. Resultados: Na amostra de 144 pacientes, $97(67,4 \%)$ apresentava idade maior que 60 anos, não havendo distinção entre os sexos. O mecanismo de trauma mais frequente foi "queda da própria altura", apresentado por $94(65,3 \%)$ pacientes. O tipo de fratura mais comum foi a de fêmur proximal, com $95(66 \%)$ casos. Conclusão: Observou-se que as fraturas de terço proximal, ocorreram em pacientes idosos, com predominância do sexo masculino nas fraturas do colo e do sexo feminino nas fraturas trocantéricas.

Palavras-chave: Epidemiologia, Fratura femoral, Internação hospitalar.

\begin{abstract}
Objective: To analyze the epidemiological, sociodemographic and clinical profile of hip and femoral fractures of patients admitted to a Hospital in the North of Minas Gerais. Methods: This is an epidemiological study, with documentary, retrospective, descriptive and analytical design conducted with 144 patients who were admitted to the hospital with a hip and femur fracture between January 2018 and January 2020. To obtain the data, a sociodemographic form (age and sex) and medical records for evolution (retrospective) were used. The data were analyzed using descriptive statistics using the IBM SPSS Statistics program. The project was approved by the Research Ethics Committee. Results: In the sample of 144 patients, $97(67.4 \%)$ were older than 60 years, with no distinction between genders. The most frequent trauma mechanism was "falling from one's height", presented by 94 (65.3\%) patients. The most common type of fracture was the proximal femur, with $95(66 \%)$ cases. Conclusion: It was observed that fractures of the proximal third occurred in elderly patients, with a predominance of males in cervical fractures and females in trochanteric fractures.
\end{abstract}

Keywords: Epidemiology, Femoral fracture, Hospitalization.

\section{RESUMEN}

Objetivo: Analizar el perfil epidemiológico, sociodemográfico y clínico de las fracturas de cadera y fémur de pacientes ingresados en un Hospital del Norte de Minas Gerais. Métodos: Se trata de un estudio epidemiológico, con diseño documental, retrospectivo, descriptivo y analítico realizado con 144 pacientes que ingresaron al hospital por fractura de cadera y fémur entre enero de 2018 y enero de 2020. Para obtener los

${ }^{1}$ Hospital Aroldo Tourinho, Montes Claros - MG.

*E-mail: rafaeloliveirarocha@yahoo.com.br

2 Universidade Estadual de Montes Claros (UNIMONTES), Montes Claros - MG.

SUBMETIDO EM: 10/2020

ACEITO EM: 11/2020

PUBLICADO EM: 12/2020

REAS/EJCH | Vol.12(12) | e5753 | DOI: https://doi.org/10.25248/reas.e5753.2020 Página 1 de 9 
datos, se utilizó un formulario sociodemográfico (edad y sexo) y la historia clínica de evolución (retrospectiva). Los datos se analizaron mediante estadística descriptiva utilizando el programa IBM SPSS Statistics. EI proyecto fue aprobado por el Comité de Ética en Investigación. Resultados: En la muestra de 144 pacientes, $97(67,4 \%)$ tenían más de 60 años, sin distinción de género. El mecanismo traumático más frecuente fue la "caída desde la altura", presentado por $94(65,3 \%)$ pacientes. El tipo de fractura más común fue el fémur proximal, con $95(66 \%)$ casos. Conclusión: se observó que las fracturas del tercio proximal ocurrieron en pacientes ancianos, con predominio del sexo masculino en las fracturas de cuello y del sexo femenino en las fracturas trocantéreas.

Palabras clave: Epidemiología, Fracturas del fémur, Hospitalización.

\section{INTRODUÇÃO}

A inversão da pirâmide demográfica, tendendo para o aumento do percentual de idosos na população, é uma realidade no Brasil, trazendo à tona também o aumento da incidência de doenças associadas a essa faixa etária como as fraturas de fêmur proximal, responsáveis pelo declínio funcional dos acometidos. Grande parte desses pacientes vai a óbito em até dois anos e muitos jamais recuperam sua qualidade de vida ou independência funcional (DANIACHI D, et al., 2015).

Além da vulnerabilidade biológica que acomete grande parte dos pacientes com lesões do fêmur proximal, outro ponto que chama atenção é a vulnerabilidade social. Nesse cenário, aspectos como escolaridade, renda per capita relações familiares e situações ambientais são responsáveis por esse tipo de vulnerabilidade e influenciam a epidemiologia dessas fraturas. Segundo Rocha L, et al. (2010), pacientes com baixa escolaridade e precariedade de renda apresentam prejuízos na manutenção de condição de saúde. Ademais, a família é a principal fonte de apoio dos idosos, tendo sua parcela de responsabilidade em salvaguardar o bem estar desses pacientes. Por último, as situações ambientais, aqui, referem-se às condições que proporcionaram a queda da própria altura desse idoso e, consequentemente, a fratura femoral. Rocha $L$, et al. (2010) traz situações como uso de calçados inadequados, presença de escadas e tapetes na moradia e cadeiras muito baixas como condições predisponentes das quedas.

Contrastando com o fator senilidade como marcador importante das fraturas proximais do fêmur, tem-se as fraturas diafisárias e distais, as quais fatores do indivíduo, como as comorbidades pouco tem influência, mas sim a energia do trauma gerador da lesão. $O$ aumento da incidência de traumas de alta energia, em que a dissipação de energia cinética é grande, vem acarretando o aparecimento de fraturas diafisárias cada vez mais graves e de difícil tratamento (PIRES RES, et al., 2010).

Além do impacto dessas lesões na qualidade de vida dos pacientes, as fraturas de fêmur de maneira geral mostram-se onerosas para os serviços públicos de saúde. Além do aumento de aproximadamente $8 \%$ no número de internações por fratura de quadril, no triênio 2006-2008, os gastos com hospitalização apenas com fraturas de fêmur proximal nos idosos foram em torno de quarenta milhões por ano, além de representarem cerca de $2 \%$ de todas as causas de hospitalização do idoso, sendo que no período de 2010-2014, o custo de tratamento das fraturas femorais foi maior que o estimado no gasto da terapêutica de neoplasias e afecções do sistema circulatório (OLIVEIRA CC e BORBA VZC, 2017).

Desse modo, a pesquisa teve como objetivo, analisar o perfil epidemiológico, sociodemográfico e clínico das fraturas de quadril e femoral de pacientes internados em um Hospital do Norte de Minas Gerais, analisando desde a etiologia das lesões, trazendo à tona os fatores de risco associados, até as formas de tratamento instituídas.

\section{MÉTODOS}

Trata-se de um estudo epidemiológico, com delineamento documental, retrospectivo, descritivo e analítico que foi realizado em um Hospital no Norte de Minas Gerais. A Instituição se destaca pela realização de atividades de ensino, pesquisa e extensão, prestando serviços na área da saúde de forma integral (100\%) pelo Sistema Único de Saúde (SUS). Na área de Urgência e Emergência, o hospital é classificado como trauma nível 2, contando com um pronto socorro 24 horas e a Ortopedia como uma das suas especialidades. 
De acordo com o programa gestão hospitalar, que consiste em um sistema informatizado de prontuários e atendimentos do hospital, 144 pacientes deram entrada no hospital com fratura de Fêmur e Quadril entre o período de janeiro de 2018 a janeiro de 2020 e compuseram a presente amostra. Após a calibração para a extração dos dados, os pesquisadores realizaram a análise dos prontuários seguindo um roteiro de dados pré-estabelecidos. Os critérios de inclusão foram: pacientes de ambos os sexos com idades a partir de 18 anos que foram submetidos ao tratamento cirúrgico. Quanto aos critérios de exclusão, dados incompletos no prontuário e pacientes que evoluíram para óbito. Para obtenção dos dados, foi utilizado um formulário sociodemográfico (idade e sexo) e prontuário para a evolução (retrospectiva) das variáveis: processo cirúrgico, processo infeccioso e as causas das fraturas.

Os dados foram analisados através da estatística descritiva (frequência simples e relativa), utilizando-se o programa Statical Package for Social Science (SPSS), versão 20.0. O projeto desse estudo foi submetido e aprovado pelo Comitê de Ética em Pesquisa da Unimontes (parecer consubstanciado 4.214.370). Essa pesquisa dispensou a utilização do Termo de Consentimento Livre e Esclarecido devido os dados serem de obtenção secundária retrospectiva de pacientes que foram atendidos ou que vieram a óbito, respeitando as diretrizes da resolução CNS Nº 466 de 2012.

\section{RESULTADOS}

De janeiro de 2018 a dezembro de 2019, foram selecionados 144 prontuários de pacientes com fratura do fêmur para esse estudo. Todos os prontuários foram analisados pelo sistema interno do hospital. Do total da amostra, $97(67,4 \%)$ pacientes apresentavam idade maior que 60 anos. Desses, $52(36,1 \%)$ estavam na faixa etária dos 61 aos 80 anos, e 45 (31,3\%) tinham mais que 80 anos de idade. Em relação ao sexo, 72 (50\%) pacientes eram do sexo masculino, e $72(50 \%)$, do sexo feminino, não havendo distinção entre os sexos nesse estudo (Tabela 1).

Tabela 1 - Número de pacientes e a porcentagem da prevalência das fraturas de fêmur de acordo com a idade e o sexo. Montes Claros - MG, 2020.

\begin{tabular}{ccc}
\hline Idade & Número de pacientes & Porcentagem \\
\hline$<18$ anos & 1 & $0,7 \%$ \\
18 a 20 anos & 5 & $3,5 \%$ \\
21 a 30 anos & 9 & $6,3 \%$ \\
31 a 40 anos & 17 & $11,8 \%$ \\
41 a 60 anos & 15 & $10,4 \%$ \\
61 a 80 anos & 52 & $36,1 \%$ \\
$>80$ anos & 45 & $31,3 \%$ \\
\hline Total & 144 & $100 \%$ \\
\hline Sexo & & \\
\hline Masculino & 72 & $50 \%$ \\
Feminino & 72 & $50 \%$ \\
\hline Total & 144 & $100 \%$ \\
\hline
\end{tabular}

Fonte: Alkmin LRF, et al., 2020. Dados extraídos do sistema de informação.

O mecanismo de trauma mais frequente que resultou na fratura foi "queda da própria altura", apresentado por $94(65,3 \%)$ pacientes. Desses, $46(31,9 \%)$ possuíam entre 61 e 80 anos de idade e $43(29,9 \%)$ eram maiores de 80 anos de idade. Apenas $5(3,5 \%)$ eram menores que 60 anos de idade. Além desses, outros mecanismos se relacionaram aos acidentes automobilísticos, sendo $26(18,1 \%)$ "acidentes de motocicleta" e $14(9,7 \%)$ "acidentes de carro". Como mecanismo também presente, porém em menor frequência, observouse "queda de altura", apresentado por 4,2\% (Tabela 2). 
Tabela 2 - Relação entre a idade e os mecanismos de trauma. Montes Claros - MG, 2020.

\begin{tabular}{|c|c|c|c|c|c|c|c|}
\hline \multirow{2}{*}{$\begin{array}{c}\text { Mecanismo } \\
\text { de } \\
\text { Trauma }\end{array}$} & \multicolumn{7}{|c|}{ Idade } \\
\hline & $\begin{array}{l}<18 \\
\text { anos }\end{array}$ & $\begin{array}{c}18 \text { a } 20 \\
\text { anos }\end{array}$ & $\begin{array}{c}21 \text { a } 30 \\
\text { anos }\end{array}$ & $\begin{array}{c}31 \text { a } 40 \\
\text { anos }\end{array}$ & $\begin{array}{c}41 \text { a } 60 \\
\text { anos }\end{array}$ & $\begin{array}{c}61 \text { a } 80 \\
\text { anos }\end{array}$ & $>80$ anos \\
\hline Queda da própria Altura & 1 & 0 & 0 & 0 & 4 & 46 & 43 \\
\hline Acidente de moto & 0 & 3 & 5 & 9 & 6 & 2 & 1 \\
\hline Acidente de carro & 0 & 2 & 2 & 4 & 3 & 2 & 1 \\
\hline Queda de altura & 0 & 0 & 1 & 2 & 1 & 2 & 0 \\
\hline Missing & 0 & 0 & 1 & 2 & 1 & 0 & 0 \\
\hline
\end{tabular}

Fonte: Alkmin LRF, et al., 2020. Dados extraídos do sistema de informação.

O tipo de fratura mais comum foi a de fêmur proximal, com 95 (66\%) casos. Dentre as fraturas de fêmur proximal, as mais frequentes foram a de colo femoral 40 (27,8\% do total de fraturas) e a transtrocantérica 39 ( $27,1 \%$ do total de fraturas). Com relação à idade e ao tipo de fratura apresentada pelos pacientes, observouse que nos pacientes acima de 60 anos houve uma maior prevalência das fraturas de fêmur proximal, já nos pacientes menores que 60 anos as fraturas diafisárias foram mais frequentes. A tabela 3 mostra a relação entre o tipo de fratura e a idade (Tabela 3).

Relacionando o mecanismo de trauma e o tipo de fratura, percebeu-se que os pacientes que tiveram "queda da própria altura" apresentaram mais fraturas do fêmur proximal. O mecanismo de trauma mais frequente das fraturas diafisária foi o "acidente de moto", com 19 (63,3\%) casos (Tabela 3).

Tabela 3 - Relação entre a idade/mecanismo de trauma e o tipo de fratura. Montes Claros - MG, 2020.

\begin{tabular}{|c|c|c|c|c|c|c|c|}
\hline \multirow[b]{2}{*}{ Idade } & \multicolumn{7}{|c|}{ Tipo de fratura } \\
\hline & $\begin{array}{c}\text { Colo } \\
\text { femoral }\end{array}$ & $\begin{array}{l}\text { Transtroc } \\
\text { antérica }\end{array}$ & $\begin{array}{c}\text { Subtrocanté } \\
\text { rica }\end{array}$ & $\begin{array}{l}\text { Basocervic } \\
\text { al }\end{array}$ & $\begin{array}{l}\text { Cabeça } \\
\text { Femoral }\end{array}$ & Diafisária & $\begin{array}{l}\text { Fêmur } \\
\text { distal }\end{array}$ \\
\hline$<18$ anos & 0 & 0 & 0 & 0 & 0 & 1 & 0 \\
\hline 18 a 20 anos & 0 & 0 & 0 & 0 & 0 & 4 & 1 \\
\hline 21 a 30 anos & 2 & 0 & 1 & 0 & 0 & 5 & 1 \\
\hline 31 a 40 anos & 0 & 1 & 2 & 0 & 0 & 11 & 2 \\
\hline 41 a 60 anos & 2 & 3 & 2 & 0 & 1 & 5 & 2 \\
\hline 61 a 80 anos & 23 & 13 & 4 & 1 & 0 & 3 & 6 \\
\hline$>80$ anos & 13 & 22 & 2 & 3 & 0 & 1 & 0 \\
\hline \multicolumn{8}{|l|}{$\begin{array}{c}\text { Mecanismo } \\
\text { de Trauma }\end{array}$} \\
\hline $\begin{array}{c}\text { Queda da } \\
\text { própria altura } \\
\text { Acidente }\end{array}$ & 36 & 36 & 5 & 4 & 0 & 2 & 5 \\
\hline $\begin{array}{l}\text { de } \\
\text { moto }\end{array}$ & 1 & 1 & 2 & 0 & 0 & 19 & 3 \\
\hline $\begin{array}{l}\text { Acidente de } \\
\quad \text { carro }\end{array}$ & 2 & 2 & 2 & 0 & 0 & 5 & 3 \\
\hline $\begin{array}{l}\text { Queda de } \\
\text { altura }\end{array}$ & 1 & 0 & 2 & 0 & 0 & 2 & 1 \\
\hline Missing & 0 & 0 & 0 & 0 & 1 & 2 & 0 \\
\hline
\end{tabular}

Fonte: Alkmin LRF, et al., 2020. Dados extraídos do sistema de informação.

Ao se relacionar o sexo e o tipo de fratura e a raça também pelo tipo, foi possível se observar que tanto os pacientes masculinos como femininos tiveram maior prevalência de fraturas do fêmur proximal do que fraturas diafisárias e distais. Quanto à raça, verificou-se que indivíduos brancos tiverem mais fraturas 
diafisárias (16 casos) e transtrocantéricas (13 casos). Já os indivíduos pardos tiveram maior prevalência de fratura transtrocantérica (21 casos) e do colo femoral (19 casos) (Tabela 4$)$.

Tabela 4 - Relação entre sexo e o tipo de fratura, e entre raça e o tipo de fratura. Montes Claros - MG, 2020.

\begin{tabular}{lccccc}
\hline \multirow{2}{*}{ Tipo de Fratura } & \multicolumn{2}{c}{ Sexo } & \multicolumn{3}{c}{ Raça } \\
\cline { 2 - 6 } & Masculino & Feminino & Branco & Pardo & Negro \\
\hline Colo femoral & 22 & 18 & 10 & 19 & 11 \\
Transtrocantérica & 18 & 21 & 13 & 21 & 5 \\
Subtrocantérica & 5 & 6 & 5 & 1 & 5 \\
Basocervical & 1 & 3 & 2 & 2 & 0 \\
Cabeça femoral & 1 & 0 & 0 & 1 & 0 \\
Diafisária & 18 & 12 & 16 & 11 & 3 \\
Fêmur distal & 5 & 7 & 1 & 9 & 2 \\
\hline
\end{tabular}

Fonte: Alkmin LRF, et al., 2020. Dados extraídos do sistema de informação.

Em relação às comorbidades prévias apresentadas pelos pacientes, verificou-se que 85 (59\%) pacientes possuíam pelo menos uma comorbidade prévia. Dentre elas, a mais frequente foi a Hipertensão Arterial Sistêmica, com 58 casos, seguida de Diabetes, Doença Neurodegenerativa, Osteoporose e Cardiopatia (com 24, 23, 20 e 19 casos respectivamente). Nesse contexto, também se identificou que os pacientes acima de 60 anos de idades foram os que mais apresentavam comorbidades, sendo que $79(81,4 \%)$ deles tinham pelo menos uma comorbidade prévia (Tabela 5).

Tabela 5 - Relação entre a idade e as comorbidades prévias. Montes Claros - MG, 2020.

\begin{tabular}{|c|c|c|c|c|c|c|c|}
\hline \multirow[b]{2}{*}{ Comorbidades } & \multicolumn{7}{|c|}{ Idade } \\
\hline & $\begin{array}{l}<18 \\
\text { anos }\end{array}$ & $\begin{array}{c}18 a \\
20 \text { anos }\end{array}$ & $\begin{array}{c}21 \text { a } \\
30 \text { anos }\end{array}$ & $\begin{array}{c}31 \text { a } \\
40 \text { anos }\end{array}$ & $\begin{array}{c}41 \text { a } \\
60 \text { anos }\end{array}$ & $\begin{array}{c}61 \text { a } \\
80 \text { anos }\end{array}$ & $\begin{array}{l}>80 \\
\text { anos }\end{array}$ \\
\hline $\begin{array}{l}\text { Hipertensão } \\
\text { Arterial } \\
\text { Sistêmica }\end{array}$ & $\begin{array}{c}0 \\
0 \%\end{array}$ & $\begin{array}{c}0 \\
0 \%\end{array}$ & $\begin{array}{c}0 \\
0 \%\end{array}$ & $\begin{array}{c}0 \\
0 \%\end{array}$ & $\begin{array}{c}3 \\
5,2 \%\end{array}$ & $\begin{array}{c}23 \\
39,7 \%\end{array}$ & $\begin{array}{c}32 \\
55,2 \%\end{array}$ \\
\hline Diabetes & $\begin{array}{c}0 \\
0 \% \\
\end{array}$ & $\begin{array}{c}0 \\
0 \% \\
\end{array}$ & $\begin{array}{c}0 \\
0 \% \\
\end{array}$ & $\begin{array}{c}0 \\
0 \% \\
\end{array}$ & $\begin{array}{c}0 \\
0 \%\end{array}$ & $\begin{array}{c}13 \\
54,2 \%\end{array}$ & $\begin{array}{c}11 \\
45,8 \% \\
\end{array}$ \\
\hline $\begin{array}{l}\text { Doença } \\
\text { Neurodegenerativa }\end{array}$ & $\begin{array}{c}0 \\
0 \%\end{array}$ & $\begin{array}{c}0 \\
0 \%\end{array}$ & $\begin{array}{c}0 \\
0 \%\end{array}$ & $\begin{array}{c}0 \\
0 \%\end{array}$ & $\begin{array}{c}1 \\
4,3 \% \\
\end{array}$ & $\begin{array}{c}8 \\
34,8 \% \\
\end{array}$ & $\begin{array}{c}14 \\
60,9 \%\end{array}$ \\
\hline Osteoporose & $\begin{array}{c}0 \\
0 \%\end{array}$ & $\begin{array}{c}0 \\
0 \%\end{array}$ & $\begin{array}{c}0 \\
0 \%\end{array}$ & $\begin{array}{c}0 \\
0 \%\end{array}$ & $\begin{array}{c}2 \\
10 \% \\
\end{array}$ & $\begin{array}{c}9 \\
45 \% \\
\end{array}$ & $\begin{array}{c}9 \\
45 \% \\
\end{array}$ \\
\hline Cardiopatia & $\begin{array}{c}0 \\
0 \%\end{array}$ & $\begin{array}{c}0 \\
0 \%\end{array}$ & $\begin{array}{c}0 \\
0 \%\end{array}$ & $\begin{array}{c}0 \\
0 \%\end{array}$ & $\begin{array}{c}1 \\
5,3 \% \\
\end{array}$ & $\begin{array}{c}7 \\
36,8 \% \\
\end{array}$ & $\begin{array}{c}11 \\
57,9 \% \\
\end{array}$ \\
\hline Doença Pulmonar & $\begin{array}{c}0 \\
0 \%\end{array}$ & $\begin{array}{c}0 \\
0 \%\end{array}$ & $\begin{array}{c}0 \\
0 \%\end{array}$ & $\begin{array}{c}0 \\
0 \%\end{array}$ & $\begin{array}{c}0 \\
0 \% \\
\end{array}$ & $\begin{array}{c}2 \\
100 \% \\
\end{array}$ & $\begin{array}{c}0 \\
0 \%\end{array}$ \\
\hline $\begin{array}{l}\text { Disfunção } \\
\text { Tireoidiana }\end{array}$ & $\begin{array}{c}0 \\
0 \%\end{array}$ & $\begin{array}{c}0 \\
0 \%\end{array}$ & $\begin{array}{c}0 \\
0 \%\end{array}$ & $\begin{array}{c}0 \\
0 \%\end{array}$ & $\begin{array}{c}0 \\
0 \%\end{array}$ & $\begin{array}{c}1 \\
50 \%\end{array}$ & $\begin{array}{c}1 \\
50 \% \\
\end{array}$ \\
\hline Mieloma Múltiplo & $\begin{array}{c}0 \\
0 \%\end{array}$ & $\begin{array}{c}0 \\
0 \%\end{array}$ & $\begin{array}{c}0 \\
0 \%\end{array}$ & $\begin{array}{c}0 \\
0 \%\end{array}$ & $\begin{array}{c}0 \\
0 \%\end{array}$ & $\begin{array}{c}1 \\
100 \%\end{array}$ & $\begin{array}{c}0 \\
0 \%\end{array}$ \\
\hline Sem Comorbidades & $\begin{array}{c}1 \\
1,7 \%\end{array}$ & $\begin{array}{c}5 \\
8,5 \%\end{array}$ & $\begin{array}{c}9 \\
15,3 \% \\
\end{array}$ & $\begin{array}{c}17 \\
28,8 \% \\
\end{array}$ & $\begin{array}{c}9 \\
15,3 \% \\
\end{array}$ & $\begin{array}{c}13 \\
22 \%\end{array}$ & $\begin{array}{c}5 \\
8,5 \%\end{array}$ \\
\hline
\end{tabular}

Fonte: Alkmin LRF, et al., 2020. Dados extraídos do sistema de informação.

De acordo com os dados, pacientes com Hipertensão Arterial Sistêmica fraturaram principalmente o fêmur proximal, sendo a fratura transtrocantérica (22 casos) e a do colo (18 casos) as mais frequentes. Sobre os pacientes com Osteoporose, também se verificou uma prevalência de fraturas no fêmur proximal, sendo as mais comuns as fraturas do colo e transtrocantérica.

Já indivíduos sem comorbidades, verificou-se uma prevalência de fraturas diafisárias, com 27 (47,4\%) casos. Quanto ao tratamento realizado, observou-se que nas fraturas diafisárias foi utilizado principalmente haste intramedular; nas fraturas de colo parafuso canulado; e nas fraturas transtrocantéricas DHS (Tabela 6). 
Tabela 6 - Relação entre o tipo de fratura e comorbidades prévias, e entre o tipo de fratura e o procedimento realizado. Montes Claros - MG, 2020.

\begin{tabular}{|c|c|c|c|c|c|c|c|c|}
\hline \multirow[b]{2}{*}{ Comorbidades } & \multicolumn{8}{|c|}{ Tipo de fratura } \\
\hline & Colo & Transt & Subtroc & Basocervical & $\begin{array}{l}\text { Cabeça } \\
\text { Femoral }\end{array}$ & Diafisária & $\begin{array}{l}\text { Fêmur } \\
\text { Distal }\end{array}$ & Total $^{*}$ \\
\hline Hipertensão & & & & & & & & \\
\hline $\begin{array}{l}\text { Arterial } \\
\text { Sistêmica }\end{array}$ & 18 & 22 & 5 & 2 & 0 & 2 & 4 & 53 \\
\hline Diabetes & 7 & 6 & 3 & 1 & 0 & 0 & 5 & 22 \\
\hline $\begin{array}{c}\text { Doença } \\
\text { Neurodegenerat } \\
\text { iva }\end{array}$ & 10 & 9 & 1 & 1 & 0 & 0 & 1 & 22 \\
\hline Osteoporose & 9 & 7 & 0 & 0 & 0 & 1 & 1 & 18 \\
\hline Cardiopatia & 5 & 9 & 2 & 0 & 0 & 1 & 1 & 18 \\
\hline $\begin{array}{l}\text { Doença } \\
\text { Pulmonar }\end{array}$ & 0 & 1 & 0 & 1 & 0 & 0 & 0 & 2 \\
\hline $\begin{array}{l}\text { Disfunção } \\
\text { Tireoidiana }\end{array}$ & 2 & 0 & 0 & 0 & 0 & 0 & 0 & 2 \\
\hline $\begin{array}{c}\text { Mieloma } \\
\text { Múltiplo } \\
\end{array}$ & 0 & 0 & 0 & 0 & 0 & & 0 & 1 \\
\hline $\begin{array}{c}\text { Sem } \\
\text { comorbidades }\end{array}$ & 10 & 9 & 5 & 0 & 1 & 27 & 5 & 57 \\
\hline $\begin{array}{c}\text { Tipo de } \\
\text { procedimento } \\
\text { submetido }\end{array}$ & & & & & & & & \\
\hline $\begin{array}{c}\text { Haste } \\
\text { Intramedular }\end{array}$ & 0 & 0 & 10 & 0 & 0 & 28 & 0 & 38 \\
\hline DHS & 0 & 32 & 0 & 3 & 0 & 0 & 0 & 35 \\
\hline $\begin{array}{l}\text { Parafuso } \\
\text { Canulado } \\
\end{array}$ & 23 & 0 & 0 & 0 & 0 & 0 & 4 & 27 \\
\hline $\begin{array}{c}\text { Artroplastia de } \\
\text { Quadril Total }\end{array}$ & 9 & 0 & 0 & 0 & 0 & 0 & 0 & 9 \\
\hline $\begin{array}{l}\text { Artroplastia de } \\
\text { Quadril Parcial }\end{array}$ & 7 & 1 & 0 & 1 & 0 & 0 & 0 & 9 \\
\hline Reconstrução & 0 & 0 & 0 & 0 & 0 & 0 & 1 & 1 \\
\hline Fixador & 0 & 0 & 0 & 0 & 0 & 0 & 1 & 1 \\
\hline Ressecção da & & & & & & & & \\
\hline $\begin{array}{c}\text { Cabeça do } \\
\text { Fêmur }\end{array}$ & 0 & 0 & 0 & 0 & 1 & 0 & 0 & 1 \\
\hline DCP & 0 & 0 & 0 & 0 & 0 & 0 & 6 & 6 \\
\hline PFN & 1 & 6 & 1 & 0 & 0 & 0 & 0 & 8 \\
\hline
\end{tabular}

Legenda: * Considerar que alguns prontuários (7) não tinham informação sobre o tipo de fratura apresentado. Fonte: Alkmin LRF, et al., 2020. Dados extraídos do sistema de informação.

\section{DISCUSSÃo}

Nesse estudo, a análise epidemiológica das lesões femorais foi ampliada para além das fraturas de terço proximal, englobando também as lesões diafisárias e do terço distal. A avaliação da amostra obtida permite a distinção entre dois perfis de pacientes acometidos pela fratura femoral, um dos perfis trata-se dos indivíduos adultos, de idade entre 21-60 anos, sem distinção de sexo, cuja etiologia da fratura decorre de traumas de alta energia, como os acidentes envolvendo carros e motocicletas, onde as comorbidades não tiveram papel de destaque.

O outro perfil trata-se de pacientes, com idade $<60$ anos, acometidos por mecanismos traumáticos de baixa energia, onde as comorbidades relatadas impactaram em todo o processo do adoecer, desde fator desencadeador do mecanismo de trauma até a escolha de tratamento. 
O traçado dos perfis epidemiológicos de cada fratura partiu da correlação entre a localização anatômica da lesão, etiologia e os dados dos indivíduos da amostra, desde a identificação com idade, sexo até a história patológica pregressa, tendo como base suas comorbidades, revelando, por exemplo, o padrão de indivíduo que busca o serviço médico em decorrência de determinada fratura.

Iniciando então pelas fraturas de terço proximal do fêmur, foi realizada a divisão dos subtipos de lesões do terço proximal, uma vez que cada uma delas correlaciona-se com um tipo de paciente. Ademais, as fraturas do colo também foram subdivididas em fraturas do colo intracapsulares e extracapsulares (basocervical). As fraturas do colo intracapsulares foram as mais comuns registradas no período da pesquisa, sendo responsável por $27,8 \%$ de todas as fraturas de fêmur. Os pacientes portadores dessa lesão encontram-se, principalmente, na faixa etária de 61-80 anos, mais jovens que os pacientes com fratura trocantérica, com pequena predominância do sexo masculino, indo de encontro com a estatística da predominância no sexo feminino nessa lesão conforme descrito na literatura (KÖBERLE G, 2001).

Dentre as comorbidades desses pacientes, destacam-se a hipertensão Arterial sistêmica (HAS), o Diabetes Mellitus (DM), a osteoporose, as cardiopatias de etiologia diversificada e as doenças neurodegenerativas, cuja distribuição encontra por faixa etária encontra-se na Tabela 6.

A presença de três ou mais comorbidades é o maior fator de risco para fraturas como fragilidade óssea prévia além de uma maior taxa de mortalidade pós-operatória. Além disso, 17,5\% deles não apresentavam qualquer comorbidade. A análise da história patológica pregressa desses indivíduos propicia a compreensão do mecanismo de trauma dessa fratura, haja vista a intima relação entre a etiologia das lesões e as patologias crônicas desses pacientes (CARVALHO MI, 2006).

O processo de senilidade, em grande partes dos casos, cursa com deficiências neuromusculares; redução dos reflexos; decréscimo da massa corporal, fatores esse que catalisam a queda da própria altura, seja pela incapacidade de evitar a queda em decorrência das deficiências neuromusculares, agravadas nos pacientes com doenças neuromusculares, e redução dos reflexos, ou mesmo pela declínio de massa corporal, que além de contribuir com a postura e equilíbrio, essa é responsável pela qualidade e proteção das estruturas ósseas (CARVALHO MI, 2006).

Outra situação bastante prosaica dentre os pacientes com fratura de colo intracapsular é predominância das doenças cardiovasculares, destacando-se a HAS como a principal comorbidade dentre elas. Os fármacos utilizados para tratamento dessa afecção facilitam a ocorrência das quedas nesses indivíduos, isso porque efeitos colaterais como incontinência urinária levam o idoso se levantar várias vezes para ir ao banheiro, principalmente à noite, em meio as armadilhas de um cenário de luminosidade, propiciando a queda.

Esse fato é evidenciado na literatura e descrito como uma das principais causas de fraturas de terço proximal atendidos nos serviços de urgência no período noturno. Por si só, o percentual de vezes que o idoso precisa se levantar e se locomover relaciona-se ao risco de queda, entretanto, episódios de hipotensão postural ocasionados por vários dos medicamentos utilizados no tratamento das HAS aumentam ainda mais a chance da queda da própria altura (SAKAKI MH, et al., 2004).

Quando ocorre a queda do paciente ao solo, o principal fator determinante na ocorrência ou não de uma fratura é a qualidade óssea. Nesse ponto o sexo do paciente apresenta destaque. Após a menopausa, as mulheres tendem a baixos níveis de estrogênio, responsável pela manutenção da massa óssea, tornando os ossos menos resistentes a impactos. (HUNGRIA NJS, et al., 2011).

Ocorrendo a fratura, o próximo passo trata-se da avaliação das opções terapêuticas para o paciente. Nesse cenário, mais uma vez as comorbidades ganham papel de destaque, uma vez que as afecções previas do paciente influenciam na escolha do tratamento e no seu prognóstico. A escolha do tratamento no serviço base da pesquisa, utiliza como parâmetro as recomendações da Sociedade Brasileira de Quadril (SBQ), que preconiza a expectativa de vida do paciente e a presença de comorbidades para a definição do tratamento.

Pacientes sem comorbidades e com fraturas sem desvio tenderam a ganhar fixação interna com parafusos canulados, tratamento utilizado em $58 \%$ desses pacientes; já os pacientes com comorbidades, 
independentemente do desvio da fratura, receberam artroplastia total ou até mesmo parcial como no caso dos pacientes com comorbidades ameaçadoras à vida ou com baixa expectativa de vida ( $<5$ anos).

O tratamento conservador foi adotado para apenas um dos pacientes do estudo. Pacientes submetidos a fixação interna apresentam uma sobrevida pós-operatória um pouco maior que os pacientes com artroplastia, entretanto essa sobrevida a longo prazo se mostra ligeiramente maior nos pacientes com artroplastia total de quadril (SAKAKI MH, et al., 2004).

Contrapondo com a representatividade das fraturas de colo intracapsulares, tem-se as fraturas basocervicais, que corresponderam a 2,8\% das fraturas de fêmur, valor concordante com a literatura atual. As fraturas basocervicais são fraturas do colo, porém comportam-se como fraturas transtrocantéricas, o que dificulta a escolha da abordagem terapêutica, além do fato de serem intrinsecamente instáveis (SHARMA A, et al., 2018). Em comparação com a fratura de colo intracapsular, as fraturas extracapsulares (basocervicais) apresentam uma maior taxa de mortalidade segunda a literatura atual. Essas fraturas extracapsulares do terço proximal ocorreram geralmente em pacientes na faixa etária dos 80-90 anos e por mecanismo de baixa cinética (queda da própria altura), entretanto, aqui, nenhum dos pacientes tinha o diagnóstico de osteoporose firmado.

Já na escolha terapêutica, a literatura é controversa quanto a escolha entre parafusos canulados e Dinamic Hip Screw (DHS). No serviço, a maior parte dos pacientes, 75\%, foi tratada com DHS (SHARMA A, et al., 2018). A fratura da cabeça femoral teve uma representatividade de $0,7 \%$ dentre as fraturas, não tendo grande relevância no perfil epidemiológico traçado.

Já as fraturas transtrocantéricas ficaram na segunda posição dentre as fraturas mais comuns no período escolhido para a coleta da amostra, representando $27,1 \%$ de todas as fraturas de fêmur. A faixa etária dessas lesões é de aproximadamente 80 anos com predominância no sexo feminino semelhante ao que é descrito nas bases de dados. Nessas lesões, as comorbidades apresentaram influencia semelhante às das fraturas de colo intracapsular, porém o fator fragilidade óssea ganha ainda mais espaço.

As fraturas de aspecto patológico associadas ganharam destaque segundo a literatura, embora 0 percentual de lesões associadas decorrente de baixa reserva óssea tenha sido irrisória neste estudo. As fraturas pertrocantéricas do estudo em geral eram estáveis, sendo tratadas, então, com DHS em $91 \%$ dos casos (KÖBERLE G, 2001).

As fraturas subtrocantéricas, conforme descrito nas referências base, representaram uma transição entre as lesões decorrentes de fragilidade óssea e uso de bifosfonados em indivíduos senis de idade superior a 60 anos e as lesões em pacientes jovens, decorrentes de traumas de alta cinética, tendo representatividade nesses dois perfis (PIRES RES, et al., 2010).

Na presente amostra também se observou um pico bimodal, sendo que $45 \%$ dos pacientes apresentavam idade menor que 60 anos, e 100\% desses, fraturaram a região subtrocantérica ocorreram em decorrência de um trauma de baixa energia. Um ponto que chama atenção no estudo é a divergência entre a representatividade numérica das fraturas subtrocantéricas em meio as fraturas de terço proximal femoral, não atingindo nem mesmo um percentual de $25 \%$ dentre as lesões do quadril conforme é descrito na literatura (LOURENÇO PRBT e PIRES RES, 2015).

O restante da amostra, com fratura subtrocantérica apresentava idade $<60$ anos e obtiveram a lesão a partir de traumas de alta cinética. Entretanto, nenhum dos 11 pacientes apresentaram histórico de osteoporose, o que pode sugerir subdiagnósticos desta afecção nesse grupo.

Essas fraturas na topografia subtrocantérica costumam ser de difícil fixação, devido a imponência das forças deformantes nessa região (SIDDHARTH BJ, et al., 2015). No serviço base a escolha foi o tratamento cirúrgico com haste centromedular para osteossíntese na maior parte dos casos, em concordância com a preferência da maioria dos autores das bases de dados (BORENS O, et al., 2004).

$\mathrm{Na}$ contramão do traçado epidemiológico das fraturas do terço proximal, as fraturas diafisárias são mais determinadas pela biomecânica de trauma do que pelo histórico de comorbidades do paciente em si. Embora seja descrito na literatura (MOARES FB, et al., 2009) uma distribuição bimodal, na amostra coletada, o perfil 
característico obtido trata-se de adultos, predominantemente do sexo masculino, cuja etiologia da fratura decorre de um trauma de alta cinética. As fraturas diafisárias de fêmur, embora potencialmente ameaçadoras a vida, tendo em vista seu potencial hemorrágico e embólico (FILOMENO LTB, et al., 2005), não geraram instabilidade hemodinâmica em nenhum dos pacientes do estudo. Todos os pacientes foram submetidos a tração transcutânea e, em segunda instância, realizado osteossíntese com haste centromedular.

Semelhante as fraturas diafisárias, as fraturas de fêmur distal apresentaram pico bimodal descrito nas bases de dados (BORGES AEA, 2012). Na amostra colhida, o mecanismo de trauma, sexo e idade apresentaram distribuição semelhante, indo desde os acidentes automobilísticos até os traumas de baixa energia, facilitados pela baixa qualidade óssea. Assim como as fraturas diafisárias, as fraturas de fêmur distal tiveram pouca influência causal das comorbidades. Há alguns anos, o tratamento conservador poderia ser uma boa opção para esse tipo de lesão, entretanto, visando melhores parâmetros de alinhamento e consolidação, o tratamento cirúrgico é o principal método utilizado nesse tipo de lesão.

\section{CONCLUSÃO}

Os pacientes admitidos no serviço base do estudo apresentaram um perfil epidemiológico que vai ao encontro da literatura disponível sobre a temática. Observou-se que as fraturas de terço proximal ocorreram em pacientes idosos, com predominância do sexo masculino nas fraturas do colo e do sexo feminino nas fraturas trocantéricas e apresentando comorbidades como cofator desencadeador de tal afecção. Os resultados enfatizaram um contraste com as lesões diafisárias e distais, presentes principalmente nos pacientes mais jovens do sexo masculino, em que a alta cinética dos traumas apresentou-se como etiologia chave. As opções terapêuticas também se concatenaram com as principais atualizações científicas.

\section{REFERÊNCIAS}

1. BORENS O, et al. Long gamma nail in the treatment of subtrochanteric fractures. Arch Orthop Trauma Surg., 2004; 124(7): 443-7.

2. BORGES AEA. Caracterização das Fraturas do Fêmur em Pacientes de um Hospital de Emergência e Trauma em João Pessoa-PB no Período de 2008/2009. R bras ci Saúde, 2012; 16(4): 507-516.

3. CARVALHO MI. OSTEOPOROSE: VISÃO DO ORTOPEDISTA. Rev. Bras. Ortop., 2006; 41(4): 91-7.

4. DANIACHI D, et al. Epidemiologia das fraturas do terço proximal do fêmur em pacientes idosos. Rev. Bras. Ortop., 2015; 50(4): 371-377.

5. FILOMENO LTB, et al. Embolia gordurosa: uma revisão para a prática ortopédica atual. Acta ortop. bras., 2005; 13(4): 196208.

6. GOMES LSM. Diagnóstico precoce da infecção articular periprotética do quadril - situação atual, avanços e perspectivas. Rev. Bras. Ortop., 2019; 54(4): 368-376.

7. HUNGRIA NJS, et al. Epidemiological characteristics and causes of proximal femoral fractures among the elderly. Rev. Bras. Ortop., 2011; 46(6): 660-667.

8. IACOVONE M, et al. Tratamento das fraturas cominutivas da diáfise do fêmur pela osteossíntese com pinos de Ender a foco fechado. Rev. Bras. Ortop., 1996; 31(6): 457-464.

9. IBRAHIM S, MELEPPURAM JJ. Uma análise retrospectiva de fraturas complexas do fêmur proximal tratadas cirurgicamente com placa de compressão bloqueada do fêmur proximal. Rev. Bras. Ortop., 2017; 52(6): 644-650.

10. KÖBERLE G. Fraturas transtrocanterianas. Rev. Bras. Ortop., 2001; 36(9): 325-329.

11. LOURENÇO PRBT, PIRES RES. Fraturas subtrocantéricas do fêmur: atualização. Rev. bras. ortop., $2016 ; 51$ (3): 246 -253.

12. MORAES FB, et al Avaliação epidemiológica e radiológica das fraturas diafisárias do fêmur: estudo de 200 casos. Rev. bras. ortop., 2009; 44(3): 199-203.

13. OLIVEIRA CC, BORBA VZC. EPIDEMIOLOGY OF FEMUR FRACTURES IN THE ELDERLY AND COST TO THE STATE OF PARANÁ, BRAZIL. Acta ortop. bras., 2017; 25(4): 155-158.

14. PIRES RES, et al. Fratura diafisária do fêmur: reprodutibilidade das classificações AO-ASIF e Winquist. Acta ortop. Bras., 2010; 18(4): 197-199.

15. ROCHA L, et al. Vulnerabilidade de idosos às quedas seguidas de fratura de quadril. Esc. Anna Nery, 2010; 14(4): 690-696.

16. ROCHE JJ, et al. Effect of comorbidities and postoperative complications on mortality after hip fracture in elderly people: prospective observational cohort study. BMJ, 2005; 331(7529):1374.

17. SAKAKI MH, et al. Estudo da mortalidade na fratura do fêmur proximal em idosos. Acta ortop. bras., 2004; $12(4): 242-249$.

18. SERFATY A. Conceitos atuais relacionados à artroplastia do quadril e suas possíveis complicações. Radiol Bras, 2020; 53(1): VII-VII.

19. SIDDHARTH BJ, et al. Contemporary Management of Subtrochanteric Fractures. Orthopedic Clinics of North America, 2015; 46(2): 21-35.

20. SHARMA A, et al. Análise comparativa do tratamento de fraturas basocervicais de fêmur com CCS, DHS e PFN em adultos jovens. Rev. Bras. Ortop., 2018; 53(6): 783-787. 